\title{
BMJ Open Clinical evaluation of enhanced recovery after surgery protocol for anterior cervical decompression and fusion (ACDF): study protocol for a multicentre randomised controlled trial
}

\author{
Gui Qin Zhong, ${ }^{1}$ Bi Hua Lin, ${ }^{1}$ Ren Qin Lin (1) , ${ }^{2}$ Ze Yan Liang, ${ }^{1}$ Yan Juan Lin, ${ }^{3}$ \\ Chun Mei Chen (D) ${ }^{2}$
}

To cite: Zhong GQ, Lin BH, Lin RQ, et al. Clinical evaluation of enhanced recovery after surgery protocol for anterior cervical decompression and fusion (ACDF): study protocol for a multicentre randomised controlled trial. BMJ Open 2021;11:e047654. doi:10.1136/ bmjopen-2020-047654

- Prepublication history and additional supplemental materia for this paper are available online. To view these files, please visit the journal online. (http://dx.doi.org/10.1136/ bmjopen-2020-047654)

GQZ and BHL contributed equally.

Received 04 December 2020 Accepted 19 July 2021

Check for updates

(c) Author(s) (or their employer(s)) 2021. Re-use permitted under CC BY-NC. No commercial re-use. See rights and permissions. Published by BMJ.

${ }^{1}$ Department of Neurosurgery, Fujian Medical University Union Hospital, Fuzhou, Fujian, China ${ }^{2}$ Department of Neurosurgery, Fujian Medical University Union Hospital PingTan Branch, Fuzhou, Fujian, China ${ }^{3}$ Nursing Department, Fujian Medical University Union Hospital, Fuzhou, Fujian, China

Correspondence to

Yan Juan Lin; fjxhlyj@163.com

\section{ABSTRACT}

Introduction To improve the efficacy of anterior cervical decompression and fusion (ACDF) and reduce postoperative complications in degenerative cervical myelopathy, our team established a set of perioperative care of enhanced recovery guidelines of ACDF based on the concept of enhanced recovery after surgery. In addition, a prospective, multicentre, randomised clinical trial was designed.

Methods and analysis A total of 260 patients aged 18-65 years will be included. Preoperative MRI and CT will be used to confirm the typical manifestations of cervical spondylosis, such as cervical disc herniation and spinal cord compression. The patient presents with neck and shoulder pain, numbness of upper limbs, weakened grip strength and cotton sense of foot tread. Patients received normal conservative treatment for 3 months with no obvious relief or even aggravation of symptoms. Patients will be assigned to the group in strict accordance with the random allocation table. Patients in groups $\mathrm{A}$ and $B$ will receive conventional perioperative care and perioperative care for enhanced recovery, respectively. The main outcome indicators are the Karnofsky Performance Scale score and the Japanese Orthopaedic Association scale. Secondary outcome indicators are pain assessment by Numeric Rating Scale, Neck Disability Index, quality of life index (QL-Index) and postoperative complications. Follow-up will be conducted at 3, 6 and 12 months postoperatively.

Ethics and dissemination Ethical approval has been granted by the Ethics Committee of Fujian Medical University Union Hospital, Fuzhou, China (2020YF034-01). Results of the research will be published in an internationa peer-reviewed scientific journal and disseminated through presentation at scientific conferences.

Trial registration number ChiCTR2000040508.

\section{INTRODUCTION}

Degenerative cervical myelopathy (DCM) is caused by direct compression of the spinal cord or inadequate local blood supply to the spinal cord. A significant aetiology is
Strengths and limitations of this study

- Large, multicentre, pragmatic, randomised controlled trial designed to evaluate the efficacy of conventional perioperative care and perioperative care of enhanced recovery in the patients of anterior cervical decompression and fusion.

- Use of standardised and validated outcome instruments.

- In different stages of this study, special personnel will be assigned to take charge of related work.

- Due to time constraints, the follow-up time of this study will be only 1 year.

cervical stenosis as it can be congenital or mostly secondary stenoses. Disc herniation, ligamentum flavum folding and osteophytes can all result in reduced cervical canal space. ${ }^{1}$ DCM is causing progressive disability and affecting the quality of life. ${ }^{23}$ The characteristic symptoms and signs of DCM include loss of flexibility of the hand, decreased muscle strength, stiffness of the limbs, urination urgency, frequency or hesitation of urination, limb spasm and gait disorder (including stiff or spastic gait). ${ }^{45}$ The conservative treatment of DCM usually includes neck support braking, analgesic drugs, epidural hormone injection, nerve root block, facet joint closure, facet joint drenching. ${ }^{5-7}$ Traction and massage may cause further compression of the spinal cord, so it should be prohibited. ${ }^{8}$ Progression of neurological dysfunction and changes in spinal cord signals on T2-weighted MRI are clear indications for surgery. The purpose of the procedure is to relieve the compression of the spinal cord and restore the normal curvature of the cervical spine.

Smith and Robinson described anterior cervical decompression and fusion (ACDF) 
in 1958. This surgical approach is still the standard surgical approach for the treatment of cervical disc herniation. ${ }^{9}$ Anterior cervical surgery, through extensive removal of the disc, can directly decompress the spinal cord and bilateral nerve roots, as well as facilitate the implantation of artificial discs or other artificial fusion materials. First proposed by Henrik Kehlet in 1997, the enhanced recovery after surgery (ERAS) is a series of perioperative optimisation measures based on evidencebased medicine to reduce the physiological and psychological traumatic stress during perioperative period, reduce complications and accelerate recovery. ${ }^{10}$ This is a multidisciplinary, multimodal approach. ${ }^{11}$ ERAS has been successfully applied in general surgery, cardiothoracic surgery, obstetrics and gynaecology, orthopaedic joint replacement surgery and other fields. ${ }^{12}$ In recent years, it has been gradually developed in the field of spinal surgery and accepted by more spinal surgeons. ${ }^{13}$ Although the treatment of DCM with ACDF has the advantages of small incision and surgical trauma, the incision is adjacent to important nerve vessels, and the tissue structure is complex, thus posing certain surgical risks and surgical complications. In addition, postoperative complications such as hoarseness and swallowing discomfort are often caused by intraoperative tractive trachea and implantation of plate internal fixation materials. ${ }^{14}$ To improve the efficacy of ACDF and reduce postoperative complications, the team established a set of perioperative care of enhanced recovery (PCER) guidelines of ACDF based on the concept of ERAS. PCER is designed to increase the perioperative nutrition intervention, preoperative intervention of early rehabilitation training, intraoperative strengthening of heat preservation, postoperative change of routine rehabilitation training mode, such as increasing the use of rehabilitation training equipment, self-made training exercises, etc., in order to promote the rapid recovery of patients after surgery, to achieve satisfactory nursing efficacy. The arising amount of data showing that the use of ERAS programmes could be helpful in reducing the days of hospitalisations and the number of complications for cervical spine surgery in a highly selected group of patients also highlight the current lack of high level of scientific evidence. ${ }^{15}$ Therefore, a prospective, multicentre, randomised clinical trial has been designed, in which patients receiving ACDF will be randomly divided into two groups and PCER and conventional perioperative care (CPC) will be applied to explore the nursing effect of PCER in patients receiving ACDF treatment.

\section{METHODS AND ANALYSIS \\ Study description}

This study is a prospective, multicentre, non-inferiority randomised controlled trial (RCT) to compare the nursing effect difference between CPC and PCER for patients treated with ACDF. The follow-up period will continue for 1 year. The actual intervention will be concealed from the subjects and follow-up researchers until the end of the follow-up period. The main assessment indicators are Karnofsky Performance Scale (KPS) score and Japanese Orthopaedic Association (JOA) score. Table 1 shows the time points of screening, randomisation and evaluation in detail.

\section{Participant recruitment and eligibility}

The participant will be conducted on a voluntary basis, so only after each appropriate patient agrees to participate and

Table 1 Flow chart visits and case report forms

\begin{tabular}{|c|c|c|c|c|c|c|}
\hline Time for evaluation & $\begin{array}{l}\text { Screening } \\
\text { period }\end{array}$ & $\begin{array}{l}\text { Intervention } \\
\text { period }\end{array}$ & $\begin{array}{l}\text { Evaluation } \\
\text { period } 1\end{array}$ & $\begin{array}{l}\text { Evaluation } \\
\text { period } 2 \\
\end{array}$ & $\begin{array}{l}\text { Evaluation } \\
\text { period } 3\end{array}$ & $\begin{array}{l}\text { Evaluation } \\
\text { period } 4\end{array}$ \\
\hline Assessment & Screen & Randomisation & $\begin{array}{l}\text { Hospital } \\
\text { discharge }\end{array}$ & $\begin{array}{l}3 \text { months } \\
\text { following } \\
\text { treatment }\end{array}$ & $\begin{array}{l}6 \text { months } \\
\text { following } \\
\text { treatment }\end{array}$ & $\begin{array}{l}12 \text { months } \\
\text { following } \\
\text { treatment }\end{array}$ \\
\hline Eligibility screen & $\sqrt{ }$ & & & & & \\
\hline Informed consent & $\sqrt{ }$ & & & & & \\
\hline Demographic information & $\sqrt{ }$ & & & & & \\
\hline Admission health education & $\sqrt{ }$ & & & & & \\
\hline Auxiliary inspection guide & $\sqrt{ }$ & $\sqrt{ }$ & $\sqrt{ }$ & $\sqrt{ }$ & $\sqrt{ }$ & $\sqrt{ }$ \\
\hline Preoperative guidance & & $\sqrt{ }$ & & & & \\
\hline Intraoperative care & & $\sqrt{ }$ & & & & \\
\hline Postoperative nursing & & $\sqrt{ }$ & $\sqrt{ }$ & & & \\
\hline Nursing document writing & $\sqrt{ }$ & $\sqrt{ }$ & $\sqrt{ }$ & & & \\
\hline Occurrence of complications & & $\sqrt{ }$ & $\sqrt{ }$ & $\sqrt{ }$ & $\sqrt{ }$ & $\sqrt{ }$ \\
\hline Discharge guidance & & & $\sqrt{ }$ & & & \\
\hline Nursing adverse events & $\sqrt{ }$ & $\sqrt{ }$ & $\sqrt{ }$ & & & \\
\hline Cervical functional assessment & $\sqrt{ }$ & & $\sqrt{ }$ & $\sqrt{ }$ & $\sqrt{ }$ & $\sqrt{ }$ \\
\hline
\end{tabular}


signs the informed consent, they will be placed on the waiting list. Detailed inclusion and exclusion criteria are as follows.

\section{Inclusion criteria}

1. Aged 18-65 years.

2. Preoperative MRI and CT confirmed signs of cervical disc herniation and compression of spinal cord.

3. Typical manifestations of cervical spondylosis, such as neck and shoulder pain, numbness of both upper limbs, weakened grip strength and cotton feeling on the feet.

4. Normal conservative treatment for 3 months, no obvious relief or even worse symptoms.

5. No absolute surgical contraindication.

6. Informed and consented.

\section{Exclusion criteria}

1. With a history of cervical spine surgery.

2. Congenital spinal stenosis or cervical deformity.

3. With dysphagia before surgery.

4. With infection and tumour.

5. Expected survival time is less than 1 year.

6 . With language communication disorder.

7. Participating in other clinical trials.

8 . With rheumatic immune diseases that may cause similar symptoms.

9. Not suitable for MRI, CT and other special examinations.

\section{Patient and public involvement statement}

The patients and the public were not (or will not) be involved in the design, or conduct, or reporting or dissemination of the research.

\section{Randomisation and blinding}

A completely randomised scheme will be used in this study. After signing the written consent, the randomisation specialist will import the patient information into the EDC system after the patient's enrolment is confirmed by the enrolment specialist. The EDC system will randomly assign the patients to PCER or CPC, and the random results will be sent to the designated mailbox. The responsible nurses will provide perioperative nursing according to the assigned results. A double-blinded design will be used in this study, for follow-up specialists and statistical specialists as well as for patients and research assistants. However, the responsible nurse has the right to know about the grouping of patients.

\section{Interventions}

Patients will be randomly assigned to the CPC and PCER groups. The common treatment measures for the two groups from admission to follow-up are as follows.

1. Health education on admission: the introduction of the chief physician and the responsible nurse; guidance on the environment, facilities and safety management of wards and advice on quitting smoking and drinking.
2. Guidance on matters requiring attention during the preoperative examination.

3. Perioperative assessment: routine nursing risk assessment, including assessment of nutritional status (NRS2002 table), inpatient fall bed/fall risk assessment, pressure ulcer risk assessment (Braden scale), thrombosis risk assessment (Caprini scale), the function of daily life activities condition assessment (Barthel table), sleep assessment (PSQI scale) and pain assessment (Numeric Rating Scale, NRS), Quality of Life Index (QL-Index). Specialist spine assessment: cervical spine dysfunction assessment (Neck Disability Index, NDI scale); neck muscle strength assessment: freehand muscle strength test (MMT), neck range of motion measurement, JOA score, KPS score, etc.

4. Preoperative preparation: cervical overextension training and guidance for the wearing of a neck brace, the handling of personal hygiene, the preparation of effective cough and sputum, and the preparation of necessary surgical items.

5. All patients will undergo ACDF under general anaesthesia.

6. Intraoperative nursing: the temperature between operations should be kept at $25^{\circ} \mathrm{C}$; the patient's body temperature will be monitored at the beginning and after the operation and catheterisation will be performed after general anaesthesia.

7. Postoperative care: the patients will be given grade I care, ECG monitoring and low-flow oxygen inhalation when they return to the ward on the day of operation. The respiratory tract will be kept unblocked and we will closely observe the changes in the patient's respiratory frequency and rhythm. Oozing fluid at the incision sight will be monitored; medical treatment will be given as advised.

8. Discharge: the patients will be given diet guidance, rest and activity guidance, discharge medication guidance, return visit guidance and rehabilitation exercise guidance.

9. Follow-up: follow-up will be conducted at 3, 6 and 12 months after surgery, including cervical spine dysfunction assessment (NDI scale), cervical mobility measurement, JOA cervical spine score, KPS functional status assessment and life quality score (QL-Index) to understand the incidence of complications.

Patients in CPC group and PCER group received different nursing programmes, as shown in the table 2.

\section{Baseline assessment}

Baseline records will include demographic, admission diagnosis, admission time, operation time, discharge time, postoperative length of stay, cost, etc.

\section{Outcomes assessment}

The following validated outcome measures will be used in the form of a questionnaire to evaluate the efficacy of nursing care. 


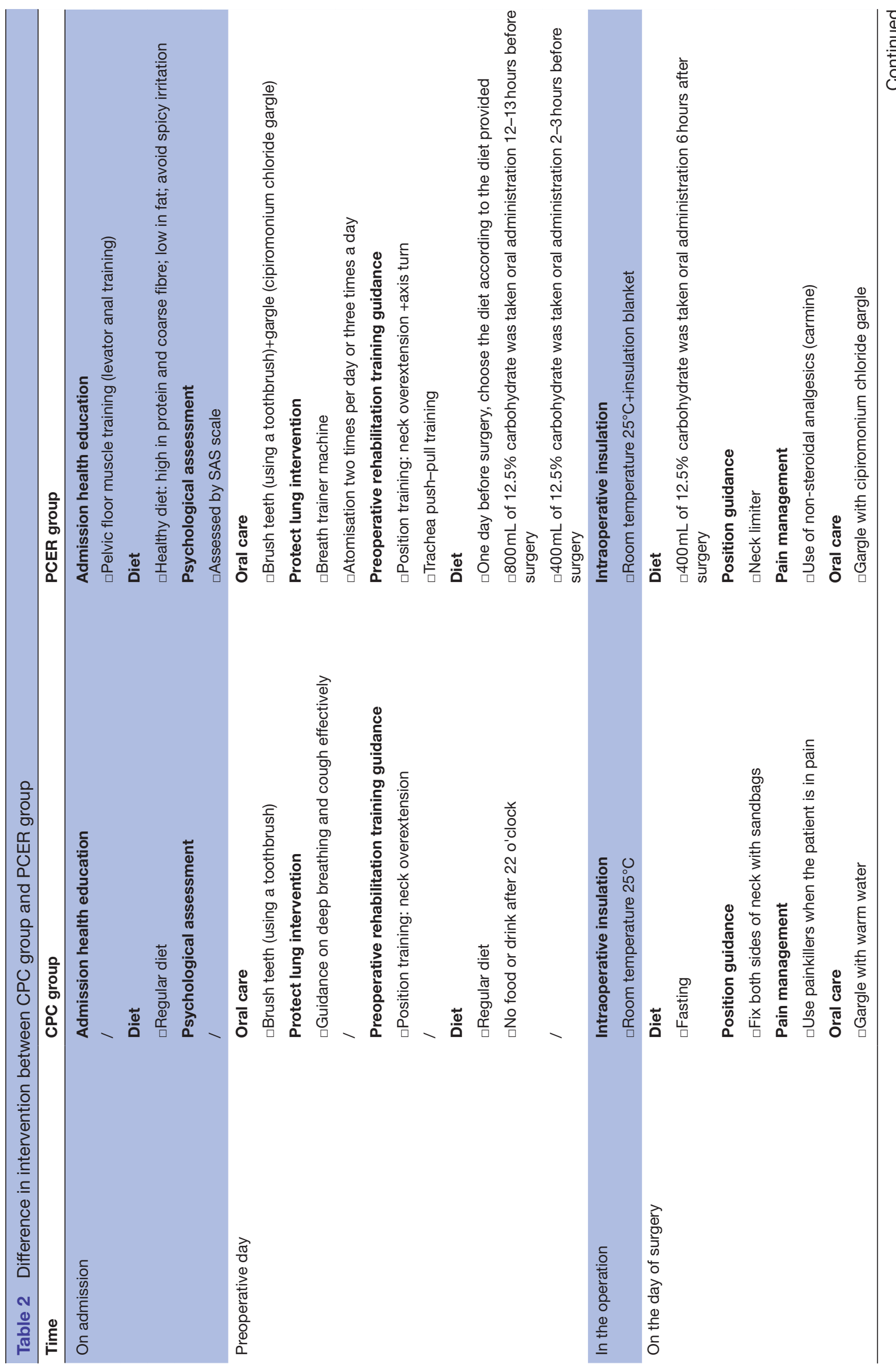

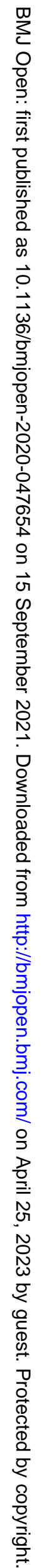




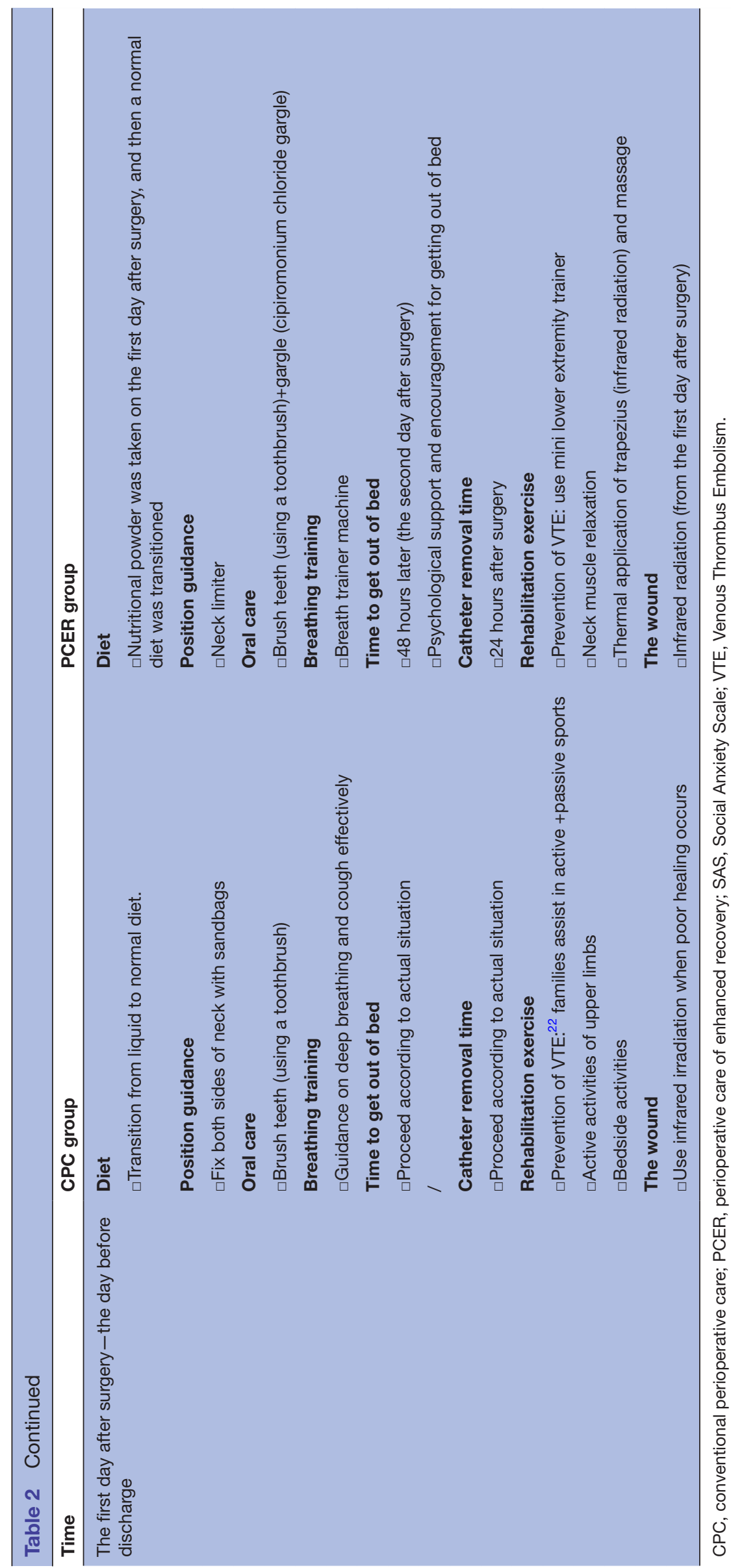

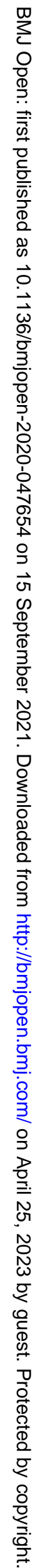




\section{Primary outcome measures}

\section{Karnofsky score (KPS) ${ }^{16}$}

The higher the KPS score, the better the health, the more tolerable the side effects of the treatment, and therefore, the more likely the patient is to undergo thorough treatment. It is generally believed that a KPS score above 80 is independent, that is, independent in daily living. A score of 50-70 is classified as semi-independent, that is, semi-independent living. A score below 50 is dependent. 2. Japanese Orthopaedic Association (JOA) ${ }^{17} 18$

The JOA scale will be used to assess neurological function. JOA scores at admission, after surgery, before discharge and during follow-up will be recorded. The full score is 17 , and the postoperative improvement rate will be calculated as follows: ( (postoperative total score-preoperative total score $) /(17$-preoperative total score $)) \times 100 \%$; improvement rate: $\geq 75 \%$, excellent; $50 \%-74 \%$, good; 25\%-49\%, medium; 0\%-24\%, poor.

\section{Secondary outcome measures}

\section{Numeric Rating Scale (NRS) ${ }^{19}$}

Pain assessment with the NRS: $0-10$ is used to represent different degrees of pain in the numeric rating method. The pain rating standard is 0 , painless; 1-3, mild pain; 4-6, moderate pain 7-10, severe pain.

2. Neck Disability Index (NDI) ${ }^{20}$

NDI is commonly used clinically to assess the cervical spine functional status. The scale includes 10 aspects: pain, personal care, lifting, reading, headache, attention, work, driving, sleep and recreation. Each item is five points and the score ranges from 0 (barrier-free) to 50 (complete paralysis); the higher the score, the worse the dysfunction. Cervical spine functional impairment index $(\%)=($ total score per project $/$ number of completed projects $\times 5) \times 100 \%$. The results were $0 \%-20 \%$, indicating mild dysfunction; $20 \%-40 \%$, indicating moderate dysfunction; $40 \%-60 \%$, severe dysfunction; $60 \%-80 \%$, very severe dysfunction and 80\%-100\%, complete dysfunction or should be carefully checked for exaggerated symptoms.

3. Quality of Life Index (QL-Index) ${ }^{21}$

QL-Index, is designed by Spitzer in 1981, is a simple scale that can quickly evaluate patients' quality of life and help estimate the treatment effect of severe diseases and the degree of disease alleviation. The scale consists of five aspects: activity, daily life, health, support and general situation, with three levels of 0.1 .2 for each item. Choose the most appropriate column item by item according to the patient's current situation, and record the corresponding score. QL-Index=Activity Score +Daily Life Score + Health Score + Support Score + General Situation Score. The total score ranged from 0 to 10 , with a high score of 10 and a low score of 0 . Those with high scores had a better quality of life than those with low scores: 0-3 was a very low score. The best was a trend analysis: an increase in scores suggested improvements in quality of life. Decreased scores indicate a deterioration in quality of life.
4. Postoperative complications

There are two main types of postoperative complications. Early surgical postoperative complications including postoperative haematoma; tissue swelling; dural tear/CSF leakage; voice hoarseness/difficulty swallowing; nerve root palsy (transient/permanent); injury to vascular structures, trachea, oesophagus and spinal cord; surgical site infection (superficial or deep). Late surgical complications including adjacent disc disease; progressive cervical kyphosis; implant pullout/subsidence; aseptic discitis and medical complications including urinary tract infection; upper respiratory tract infection; deep vein thrombosis/pulmunary embolism; myocardial infarct; stroke; death.

\section{Sample size}

In this study, the sample size was calculated according to $\alpha=0.05, \beta=0.2$, sample ratio $1: 1$, and the JOA score of 150 patients with DCM from 2017 to 2019 in our hospital at 1 year after surgery was taken into account, $\mu \mathrm{A}=15.5$, $\mu \mathrm{B}=15.1, \sigma=1.1$. A total of 95 samples were calculated for each group. When the shedding rate was set at $20 \%$, the final sample size was 238 cases. According to the actual situation of each centre, 260 subjects will be included in this study. We expect to complete subject recruitment within 2 years. Subjects will be recruited on 1 January 2021 .

\section{Statistical analysis}

Both sets of baseline data will include demographic information, general conditions prior to intervention and baseline indicators that may influence prognosis. The mean, SD and CI of the measurement data will be given, and the minimum, maximum, P25, median and $\mathrm{P} 75$ will be provided when necessary. Measurement data shall be tested by t-test or a non-parametric t-test (when variance is uneven); the Pearson2 test will be used for counting data; Wilcoxon two-sample rank sum test will be used for grade data. The Pearson2 test will be used to compare the incidence of adverse events between the two groups. A p value less than 0.05 will be considered statistically significant. The analysis will be performed using appropriate statistical software, such as SPSS.

\section{ETHICS AND DISSEMINATION}

This article describes a protocol for a prospective, noninferiority RCT to examine the efficacy of CPC and PCER in the patients of ACDF. Informed consent will be obtained prior to randomisation from all eligible participants (online supplemental appendix 1). Results of the research will be published in an international peerreviewed scientific journal and disseminated through presentation at scientific conferences.

Contributors All authors are the principal investigators and have coordinated all the phases of trial design, statistical analysis plan and drafting of the protocol. G-QZ, Y-JL and CC completed the conception and design of the project. BL and ZL carried out the acquisition of data or analysis and interpretation of data. BL, RQL 
and ZL wrote the manuscript. All authors contributed to refinement of the study protocol and approval of the final manuscript.

Funding Joint Funds for the innovation of Science and Technology, Fujian province (grant no 2018Y9060).

Competing interests None declared.

Patient consent for publication Obtained.

Provenance and peer review Not commissioned; externally peer reviewed.

Supplemental material This content has been supplied by the author(s). It has not been vetted by BMJ Publishing Group Limited (BMJ) and may not have been peer-reviewed. Any opinions or recommendations discussed are solely those of the author(s) and are not endorsed by BMJ. BMJ disclaims all liability and responsibility arising from any reliance placed on the content. Where the content includes any translated material, BMJ does not warrant the accuracy and reliability of the translations (including but not limited to local regulations, clinical guidelines, terminology, drug names and drug dosages), and is not responsible for any error and/or omissions arising from translation and adaptation or otherwise.

Open access This is an open access article distributed in accordance with the Creative Commons Attribution Non Commercial (CC BY-NC 4.0) license, which permits others to distribute, remix, adapt, build upon this work non-commercially, and license their derivative works on different terms, provided the original work is properly cited, appropriate credit is given, any changes made indicated, and the use is non-commercial. See: http://creativecommons.org/licenses/by-nc/4.0/.

ORCID iDs

Ren Qin Lin http://orcid.org/0000-0003-4479-1556

Chun Mei Chen http://orcid.org/0000-0002-4483-7465

\section{REFERENCES}

1 Parke WW. Correlative anatomy of cervical spondylotic myelopathy. Spine 1988;13:831-7.

2 Theodore N. Degenerative cervical spondylosis. N Engl J Med 2020;383:159-68.

3 King JT, McGinnis KA, Roberts MS. Quality of life assessment with the medical outcomes study short form-36 among patients with cervical spondylotic myelopathy. Neurosurgery 2003;52:113-20.

4 Denno JJ, Meadows GR. Early diagnosis of cervical spondylotic myelopathy. A useful clinical sign. Spine 1991;16:1353-5.

5 Bakhsheshian J, Mehta VA, Liu JC. Current diagnosis and management of cervical spondylotic myelopathy. Global Spine $J$ 2017;7:572-86.

6 Fehlings MG. Current knowledge in degenerative cervical myelopathy. Neurosurg Clin N Am 2018;29:xiii-xiv.

7 Ganau M, Holly LT, Mizuno J, et al. Future directions and new technologies for the management of degenerative cervical myelopathy. Neurosurg Clin N Am 2018;29:185-93.
8 Yoo JU, Zou D, Edwards WT, et al. Effect of cervical spine motion on the neuroforaminal dimensions of human cervical spine. Spine 1992;17:1131-6.

9 Smith GW, Robinson RA. The treatment of certain cervical-spine disorders by anterior removal of the intervertebral disc and interbody fusion. J Bone Joint Surg Am 1958;40-A:607-24.

10 Joliat G-R, Labgaa I, Petermann D, et al. Cost-Benefit analysis of an enhanced recovery protocol for pancreaticoduodenectomy. Br J Surg 2015;102:1676-83.

11 Kehlet $\mathrm{H}$. Multimodal approach to control postoperative pathophysiology and rehabilitation. Br J Anaesth 1997;78:606-17.

12 Nygren J, Thacker J, Carli F, et al. Guidelines for perioperative care in elective rectal/pelvic surgery: enhanced recovery after surgery (ERAS $\left.{ }^{8}\right)$ Society recommendations. Clin Nutr 2012;31:801-16.

13 Tiansheng S, Jianxiong S, Zhongjun L. Expert consensus in enhanced recovery after spinal surgery in China : perioperative management. Chinese Journal of Bone and Joint Surgery 2017;10:271-9.

14 Fountas KN, Kapsalaki EZ, Nikolakakos LG, et al. Anterior cervical discectomy and fusion associated complications. Spine 2007;32:2310-7.

15 Zaed I, Bossi B, Ganau M, et al. Current state of benefits of enhanced recovery after surgery (ERAS) in spinal surgeries: a systematic review of the literature. Neurochirurgie 2021;S00283770(21)00107-7. doi:10.1016/j.neuchi.2021.04.007. [Epub ahead of print: 23 Apr 2021].

16 Terret C, Albrand G, Moncenix G, et al. Karnofsky performance scale (KPS) or physical performance test (PPT)? that is the question. Crit Rev Oncol Hematol 2011;77:142-7.

17 Kato S, Oshima Y, Oka H, et al. Comparison of the Japanese orthopaedic association (JOA) score and modified JOA (mJOA) score for the assessment of cervical myelopathy: a multicenter observational study. PLoS One 2015;10:e0123022.

18 Martin AR, Jentzsch T, Wilson JRF, et al. Inter-Rater reliability of the modified Japanese orthopaedic association score in degenerative cervical myelopathy. Spine 2021;Publish Ahead of Print. doi:10.1097/ BRS.0000000000003956. [Epub ahead of print: 22 Jan 2021].

19 Hawker GA, Mian S, Kendzerska T, et al. Measures of adult pain: visual analog scale for pain (vas pain), numeric rating scale for pain (NRS pain), McGill pain questionnaire (MPQ), short-form McGill pain questionnaire (SF-MPQ), chronic pain grade scale (CpGs), short Form-36 bodily pain scale (SF. Arthritis Care Res 2011;63:S240-52.

20 Vernon $\mathrm{H}$, Mior S. The neck disability index: a study of reliability and validity. J Manipulative Physiol Ther 1991;14:409-15.

21 Spitzer WO, Dobson AJ, Hall J, et al. Measuring the quality of life of cancer patients. J Chronic Dis 1981;34:585-97.

22 Chibbaro S, Cebula H, Todeschi J, et al. Evolution of prophylaxis protocols for venous thromboembolism in neurosurgery: results from a prospective comparative study on low-molecular-weight heparin, elastic stockings, and intermittent pneumatic compression devices. World Neurosurg 2018;109:e510-6. 